\title{
Congruence of Patient- and Clinician-Reported Toxicity in Women Receiving Chemotherapy for Early Breast Cancer
}

\author{
Kirsten A. Nyrop, PhD (DD 1,2; Allison M. Deal²; Bryce B. Reeve (iD) 3; Ethan Basch ${ }^{1,2}$; Yi Tang Chen ${ }^{4}$; Ji Hye Park ${ }^{4}$; \\ Shlomit S. Shachar ${ }^{5}$; Lisa A. Carey ${ }^{1,2}$; Katherine E. Reeder-Hayes (iD 1,2; Elizabeth C. Dees ${ }^{1,2}$; Trevor A. Jolly ${ }^{1,2}$; \\ Gretchen G. Kimmick ${ }^{3}$; Meghan S. Karuturi (D) 6; Raquel E. Reinbolt (iD 7; JoEllen C. Speca'; Jordan T. Lee ${ }^{8}$; \\ William A. Wood ${ }^{1,2}$; and Hyman B. Muss ${ }^{1,2}$
}

\begin{abstract}
BACKGROUND: The National Cancer Institute's Patient-Reported Outcomes Version of the Common Terminology Criteria for Adverse Events, collected alongside the clinician-reported Common Terminology Criteria for Adverse Events, enables comparisons of patient and clinician reports on treatment toxicity. METHODS: In a multisite study of women receiving chemotherapy for early-stage breast cancer, symptom reports were collected on the same day from patients and their clinicians for 17 symptoms; their data were not shared with each other. The proportions of moderate, severe, or very severe patient-reported symptom severity were compared with the proportions of clinician-rated grade 2, 3, or 4 toxicity. Patient-clinician agreement was assessed via $\mathrm{k}$ statistics. Chi-square tests investigated whether patient characteristics were associated with patient-clinician agreement. RESULTS: Among 267 women, the median age was 58 years (range, $24-83$ years), and $26 \%$ were nonwhite. There was moderate scoring agreement ( $K=0.413-0.570$ ) for $53 \%$ of symptoms, fair agreement for $41 \%(\mathrm{~K}=0.220-0.378)$, and slight agreement for $6 \%(\mathrm{~K}=0.188)$. For example, patient-reported and clinician-rated percentages were $22 \%$ and $8 \%$ for severe or very severe fatigue, $41 \%$ and $46 \%$ for moderate fatigue, $32 \%$ and $39 \%$ for mild fatigue, and $6 \%$ and $7 \%$ for none. Clinician severity scores were lower for nonwhite patients in comparison with white patients for peripheral neuropathy, nausea, arthralgia, and dyspnea. CONCLUSIONS: Although clinician reporting of symptoms is common practice in oncology, there is suboptimal agreement with the gold standard of patient self-reporting. These data provide further evidence supporting the integration of patient-reported outcomes into oncological clinical research and clinical practice to improve monitoring of symptoms as well as timely interventions for symptoms. Cancer 2020;126:3084-3093. (c) 2020 American Cancer Society.
\end{abstract}

KEYWORDS: breast, chemotherapy, clinician-rated, patient-reported, symptoms.

\section{INTRODUCTION}

The National Cancer Institute's Common Terminology Criteria for Adverse Events (CTCAE) ${ }^{1}$ is the long-standing standard approach for the collection and reporting of adverse events in oncology research. ${ }^{2}$ Of the approximately 800 adverse events included in the CTCAE item library, approximately 10\% correspond to symptoms, such as nausea and sensory neuropathy. However, CTCAE items are recorded by clinical research staff rather than patients. In response to growing evidence for the value of patient-reported symptom severity as a complement to clinician-assessed toxicity, ${ }^{3}$ the National Cancer Institute supported the development of the Patient-Reported Outcomes Version of the Common Terminology Criteria for Adverse Events (PRO-CTCAE), ${ }^{4,5}$ which became publicly available in April 2016. Like CTCAE, PROCTCAE provides single-item measures for patient-reported symptom severity and also includes items for interference with usual or daily activities and the frequency of some symptoms.

The development of PRO-CTCAE held the promise of improved understanding of patient and clinician toxicity reports for multiple symptoms simultaneously and at multiple time points during chemotherapy if PRO-CTCAE

Corresponding Author: Kirsten A. Nyrop, PhD, Division of Hematology-Oncology, School of Medicine, University of North Carolina at Chapel Hill, 170 Manning Dr, Campus Box 7305, Chapel Hill, NC 27599-7305 (kirsten_nyrop@med.unc.edu).

${ }^{1}$ School of Medicine, University of North Carolina at Chapel Hill, Chapel Hill, North Carolina; ${ }^{2}$ Lineberger Comprehensive Cancer Center, University of North Carolina at Chapel Hill, Chapel Hill, North Carolina; ${ }^{3}$ Duke University School of Medicine, Durham, North Carolina; ${ }^{4}$ Gillings School of Global Public Health, University of North Carolina at Chapel Hill, Chapel Hill, North Carolina; ${ }^{5}$ Rambam Health Campus, Haifa, Israel; ${ }^{6}$ The University of Texas MD Anderson Cancer Center, University of Texas, Houston, Texas; ${ }^{7}$ Ohio State University Comprehensive Cancer Center, Columbus, Ohio; ${ }^{8}$ Department of Exercise and Sport Science, University of North Carolina at Chapel Hill, Chapel Hill, North Carolina

The ClinicalTrials.gov identifiers for the studies are NCT02167932, NCT02328313, and NCT03761706.

We greatly appreciate the active support of oncology clinicians and their research staff at multiple sites and, most importantly, the patients with breast cancer participating in our study. The sites are the Duke University Medical Center/Cancer Institute, the Ohio State University Comprehensive Cancer Center, the MD Anderson Cancer Center, University of North Carolina Rex Healthcare, and the University of North Carolina Cancer Center. We also thank Tucker Brenizer, Erin O'Hare, Nicole Markowski, Nora Christopher, Emily Bell, Chad Wagoner, Will Pulley, Nancy Burns, and Amy Garrett for their unwavering commitment to study implementation best practices.

Additional supporting information may be found in the online version of this article.

DOI: 10.1002/cncr.32898, Received: January 8, 2020; Revised: February 14, 2020; Accepted: March 15, 2020, Published online April 21, 2020 in Wiley Online Library (wileyonlinelibrary.com) 
and CTCAE reports were completed in real time during the same clinic visit. ${ }^{6}$ This would enable more rigorous analyses of convergence and divergence in patient and clinician perspectives on important clinical endpoints such as quality of life and function. ${ }^{7}$ PRO-CTCAE could also facilitate collaborative reporting on symptoms that are not asked about routinely through a process in which patient-reported toxicity forms are made readily available to the treating clinician during routine clinic visits. ${ }^{8}$

Within a growing body of literature documenting discrepancies between patient- and clinician-reported toxicities, ${ }^{8}$ studies making paired comparisons of health care provider-assessed CTCAE and patient-reported PRO-CTCAE (or patient-tested precursors to PROCTCAE) have been conducted in patients receiving chemotherapy for head and neck cancer, ${ }^{9}$ genitourinary cancer, ${ }^{10}$ and lung cancer, ${ }^{10,11}$ patients receiving radiotherapy, ${ }^{12}$ and patients receiving chemotherapy and/ or radiation therapy, ${ }^{5}$ with 4 of these studies collecting data at more than 1 time point during treatment. ${ }^{5,9,11,12}$ Additional studies have compared CTCAE toxicity grades with validated symptom measures such as the European Organisation for the Research and Treatment of Cancer Core Quality of Life Questionnaire (QLQC30 $)^{13-15}$ and other study-specific symptom reports, ${ }^{16}$ with 1 of these studies collecting data at multiple time points during treatment. ${ }^{14}$ Some of the aforementioned studies included women with early breast cancer within a mixed sample of adults with cancer; however, findings were not reported separately for each type of cancer $^{5,13,16}$ or had a specific focus on early-stage breast cancer.

In this study, we conducted an analysis among women with early-stage breast cancer in which we compared clinician-reported (CTCAE) and patient-reported (PRO-CTCAE) severity for 17 symptoms collected at multiple time points throughout chemotherapy. We have previously reported that patient-assessed symptom severity for these 17 symptoms varies significantly among 4 chemotherapy regimens commonly used in current clinical practice, ${ }^{17}$ and this confirms the importance of continuous symptom monitoring throughout treatment. We have also reported that there is minimal agreement between patient- and clinician-reported severity scores for chemotherapy-induced peripheral neuropathy associated with these chemotherapy regimens. ${ }^{18}$ In the current study, we compare patient and clinician reports for all 17 symptoms, and we identify factors that may be associated with patient-clinician consensus or divergence. ${ }^{13}$

\section{MATERIALS AND METHODS}

\section{Study Participants}

This is a secondary analysis of data from a sample of women recruited into 1 of 3 prospective, nonrandomized studies of a walking intervention for patients receiving (neo)adjuvant chemotherapy for early breast cancer (stages 0-III according to American Joint Committee on Cancer staging, 7th edition; ClinicalTrials.gov identifiers NCT02167932, NCT02328313, and NCT03761706). Patients were 21 years old or older and were recruited before starting chemotherapy regimens that were selected by clinicians in consultation with their patients. Patients provided written informed consent, and the studies were approved by the University of North Carolina Lineberger Comprehensive Cancer Center's protocol review committee and the institutional review boards for each study site.

\section{Measures}

From chemotherapy initiation through the end of chemotherapy, patients completed a patient-reported symptom form for 17 symptoms. These symptoms were selected a priori for their observed frequency in the treatment of patients with early breast cancer. In 2 studies (NCT02167932 and NCT02328313), the reporting form was the validated patient-reported symptom monitor (PRSM). ${ }^{19}$ The PRSM was a precursor to PRO-CTCAE and was used because PRO-CTCAE was not publicly available when these 2 studies were initiated. The PRSM precursor was developed by investigators who were also involved with the development of PRO-CTCAE ${ }^{4,20}$ and has a structure and response scale analogous to those of PRO-CTCAE (Supporting Table 1); it uses single-item measures of symptom severity on a 5-point scale with response options ranging from "none/no symptom" to "very severe." ${ }^{21}$ In addition, using a single-item measure, patients reported the symptom "interference with doing things you usually do" with similar 5-point response options ranging from "not at all" to "very much." When PRO-CTCAE became publicly available, it was used as the reporting form for the third study (NCT03761706). Depending on their chemotherapy infusion schedule over 4 to 8 total cycles, patients completed symptom reports every other week or every third week. Patients with weekly infusion schedules (mostly paclitaxel) completed 
symptom reports every other week to avoid overreporting in this cohort in comparison with the rest of the sample. Patients completed symptom reports during their chemotherapy infusion, which was after they had seen their oncology clinician.

On the same day that patients completed symptom reports, their oncology clinician $\mathrm{MD}$, nurse practitioner, or physician assistant) was asked to complete a CTCAE study form to rate the same set of 17 symptoms. The patient reports were not available to their clinicians. For comparison with patient-reported scores, CTCAE response options were standardized across symptoms as follows: $0=$ none, $1=$ mild, $2=$ moderate, $3=$ severe, and $4=$ disabling. ${ }^{22,23}$ We matched patient-reported "none" with CTCAE grade 0 , "mild" with grade 1, "moderate" with grade 2, and "severe/very severe" with grade $3 / 4^{21,24}$; this was consistent with a previously developed mapping algorithm. ${ }^{22,23}$

\section{Statistical Analysis}

Descriptive statistics were used to summarize patient characteristics, breast cancer diagnoses and treatments, adverse events, and patient- and clinician-reported symptom scores. Because clinicians were not always available to complete reports, only data points from days on which both the patient and the clinician reported were included. The essential metric for our study was the maximum score for each symptom at any time during the measurement period (start to end of chemotherapy); this is the approach used in clinical trials when treatment toxicity is reported. The proportions of moderate, severe, or very severe patient-reported symptom severity and interference were compared with the proportions of clinician-rated grade 2, 3, or 4 toxicity for all 17 symptoms combined and for each symptom individually.

We assessed agreement between patient- and clinician-reported dichotomized maximum scores by reporting simple $\kappa$ coefficients for each symptom. ${ }^{25}$ Dichotomization was "low" for none or mild and "high" for moderate, severe, or very severe. A priori interpretation of the $\kappa$ statistic used standard rating criteria $^{26}:<0.0$, less than chance agreement; 0.01 to 0.20 , slight agreement; 0.21 to 0.40 , fair agreement; 0.41 to 0.60 , moderate agreement; 0.61 to 0.80 , substantial agreement; and 0.81 to 0.99 , almost perfect agreement. The same method was used to compare the patientreported symptom "interference with things you usually like to do" with the clinician toxicity grade. Chi-square tests were conducted to investigate whether patient characteristics were associated with patient-clinician
TABLE 1. Study Participant Characteristics $(n=267)$

\begin{tabular}{|c|c|}
\hline Variable & Value \\
\hline Age, median (SD), y & $58(13)$ \\
\hline Age, range, y & $24-83$ \\
\hline \multicolumn{2}{|l|}{ Race, No. (\%) } \\
\hline Not white & $70(26)$ \\
\hline White & $197(74)$ \\
\hline \multicolumn{2}{|l|}{ Education, No. (\%) } \\
\hline High school or less & $38(14)$ \\
\hline More than high school & $227(86)$ \\
\hline \multicolumn{2}{|l|}{ Married, No. (\%) } \\
\hline No & $116(44)$ \\
\hline Yes & $149(56)$ \\
\hline Body mass index, mean (SD), $\mathrm{kg} / \mathrm{m}^{2}$ & $30(7)$ \\
\hline Body mass index, range, $\mathrm{kg} / \mathrm{m}^{2}$ & $17-65$ \\
\hline \multicolumn{2}{|l|}{ Body mass index, No. (\%) } \\
\hline Underweight $\left(<18.5 \mathrm{~kg} / \mathrm{m}^{2}\right)$ & $3(1)$ \\
\hline Normal $\left(18.5\right.$ to $\left.<25 \mathrm{~kg} / \mathrm{m}^{2}\right)$ & $72(27)$ \\
\hline Overweight $\left(25\right.$ to $\left.<30 \mathrm{~kg} / \mathrm{m}^{2}\right)$ & $83(31)$ \\
\hline Obese I $\left(\geq 30 \mathrm{~kg} / \mathrm{m}^{2}\right)$ & $109(41)$ \\
\hline \multicolumn{2}{|c|}{ Menopausal status at breast cancer diagnosis, No. (\%) } \\
\hline Premenopausal & $81(31)$ \\
\hline Postmenopausal & $183(69)$ \\
\hline \multicolumn{2}{|l|}{ Breast cancer stage, No. (\%) } \\
\hline I & $67(25)$ \\
\hline ॥ & $133(50)$ \\
\hline III & $67(25)$ \\
\hline \multicolumn{2}{|l|}{ Breast cancer phenotype, No. (\%) } \\
\hline HR-negative/HER2-negative & $78(29)$ \\
\hline HR-negative/HER2-positive & $34(13)$ \\
\hline HR-positive/HER2-negative & $120(45)$ \\
\hline HR-positive/HER2-positive & $34(13)$ \\
\hline \multicolumn{2}{|l|}{ Breast cancer surgery, No. (\%) } \\
\hline None & $7(3)$ \\
\hline Lumpectomy & $126(48)$ \\
\hline Mastectomy & $127(49)$ \\
\hline Anti-HER2 therapy, No. (\%) & $67(25)$ \\
\hline \multicolumn{2}{|l|}{ Chemotherapy timing, No. (\%) } \\
\hline Neoadjuvant & $103(39)$ \\
\hline Adjuvant & $159(60)$ \\
\hline Both & $1(1)$ \\
\hline \multicolumn{2}{|c|}{ Chemotherapy regimens: drug combinations, No. (\%) } \\
\hline AC-T & $82(31)$ \\
\hline AC-TC & $19(7)$ \\
\hline $\mathrm{TC}^{\mathrm{a}}$ & 70 (27) \\
\hline $\mathrm{TCH}$ & $41(16)$ \\
\hline Other & 51 (19) \\
\hline
\end{tabular}

Abbreviations: AC-T, doxorubicin and cyclophosphamide followed or preceded by paclitaxel/Taxol; AC-TC, doxorubicin and cyclophosphamide plus paclitaxel and carboplatin; TC, docetaxel and cyclophosphamide with or without anti-human epithelial growth factor receptor 2 (HER2) therapy; TCH; docetaxel and carboplatin plus anti-human epithelial growth factor receptor 2 (HER2) therapy.

${ }^{\text {a } T h r e e ~ p a t i e n t s ~ a l s o ~ r e c e i v e d ~ a n t i-H E R 2 ~ t h e r a p y . ~}$

agreement on maximum severity scores for each symptom individually.

\section{RESULTS}

\section{Patient Characteristics}

In a sample of 267 women, the median age was 58 years (range, 24-83 years), and 26\% were nonwhite. Breast cancer was distributed across stages I, II, and III and 

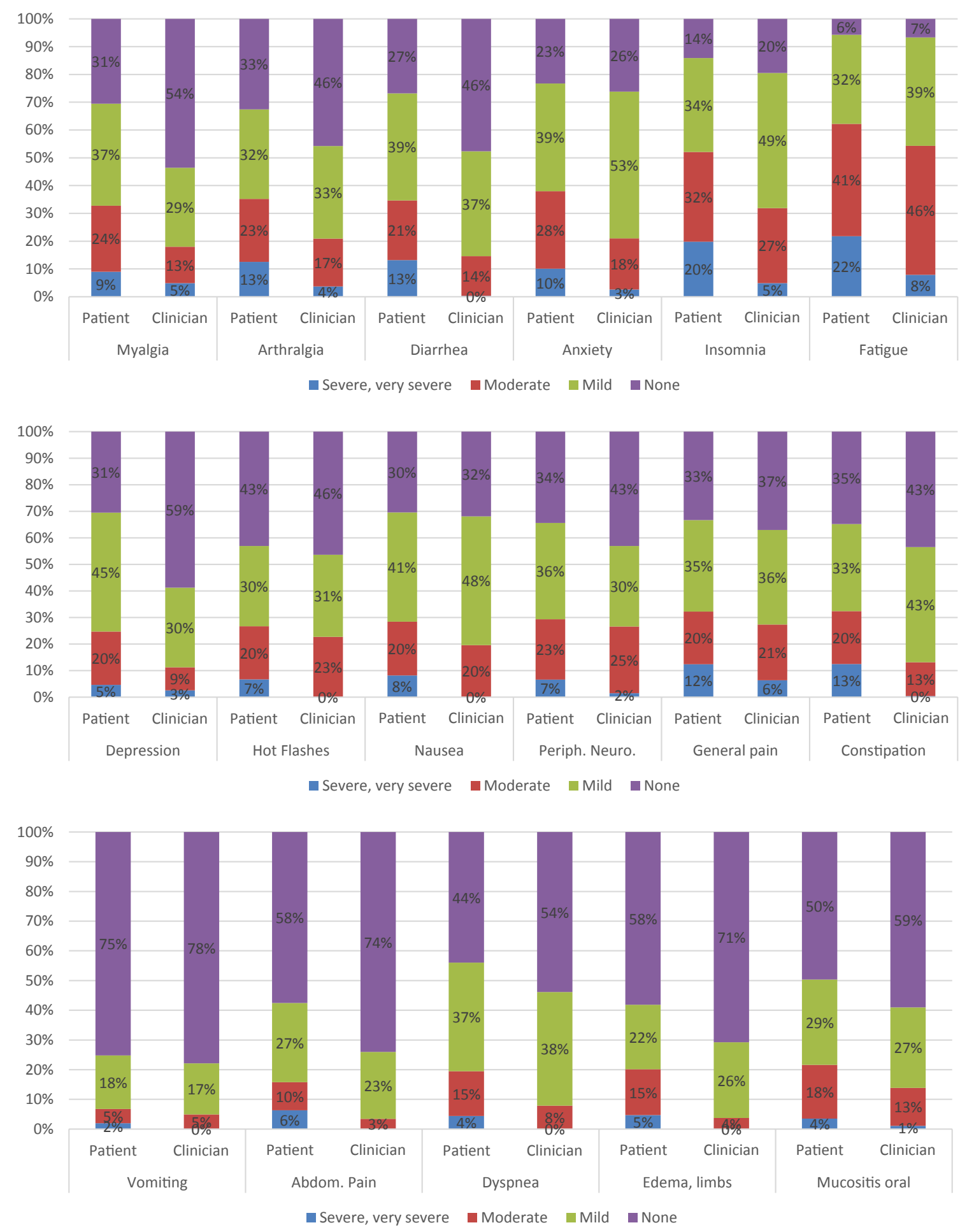

Figure 1. Maximum severity score at any time during chemotherapy: patient and clinician scores.

across 4 different common chemotherapy regimens (Table 1). A total of 1203 same-day paired reports were considered in our analysis, and the maximum symptom score for each patient was the unit of analysis.
For patients receiving doxorubicin and cyclophosphamide followed or preceded by paclitaxel/Taxol (AC-T) or doxorubicin and cyclophosphamide plus paclitaxel and carboplatin (AC-TC), the median number of 


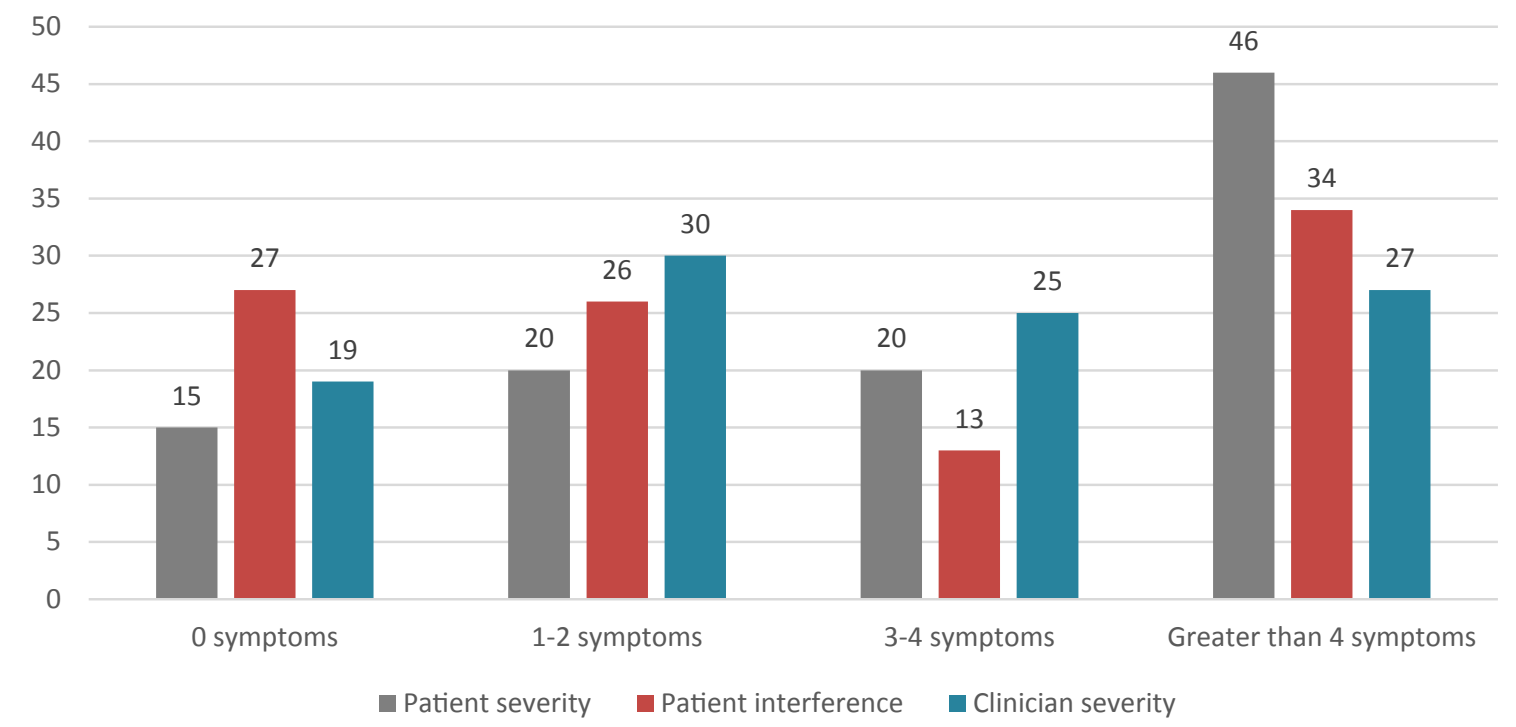

Figure 2. Patient-reported moderate, severe, or very severe symptom severity and interference and clinician-rated grade 2 , 3, or 4 toxicity (percentages of patients).

reports was 6; for those receiving docetaxel and cyclophosphamide (TC) with or without anti-human epithelial growth factor receptor 2 (HER2) therapy, it was 3; for those receiving docetaxel and carboplatin plus anti-HER2 therapy $(\mathrm{TCH})$ it was 5 ; and for all regimens combined, it was 4 .

\section{Patient and Clinician Symptom Severity Scores}

In Figure 1, the proportions of patient-reported maximum severity scores ranging from none to severe/very severe and clinician toxicity grades ranging from 0 to $3 / 4$ are presented for individual symptoms. For example, patient- and clinician-rated percentages were $22 \%$ and $8 \%$ for severe or very severe fatigue, $41 \%$ and $46 \%$ for moderate fatigue, $32 \%$ and $39 \%$ for mild fatigue, and $6 \%$ and $7 \%$ for none. This figure illustrates how the proportions of patient-reported moderate and severe/very severe symptoms were consistently higher than the proportions of clinician-rated toxicity grades 2 and 3/4.

Figure 2 illustrates the percentages of patients who rated their symptom severity or interference as moderate, severe, or very severe and the percentages of clinicians who rated their toxicity grade 2,3 , or 4 . For example, in the far right grouping, $46 \%$ of patients rated more than 4 symptoms as moderate or worse in severity; $34 \%$ of patients rated more than 4 symptoms as moderate or worse in interference; and $27 \%$ of patients were rated by their clinician as having more than 4 symptoms graded 2 , 3 , or 4 . In the far left group, $15 \%$ of patients rated none of their symptoms as moderate or worse in severity, $27 \%$ of patients rated symptom interference as moderate or worse, and $19 \%$ of patients had none of their symptoms graded by their clinicians as 2 or higher.

\section{Agreement of Patient- and Clinician-Rated Symptom Severity}

Table 2 shows the proportions of study participants for whom patients and clinicians agreed that symptom severity was low (none or mild) or high (moderate or severe/ very severe). The table also shows where clinician maximum severity scores were higher than patient scores (clinician high/patient low) and where patient maximum scores were higher than clinician scores (patient high/ clinician low). For example, for constipation, there was patient-physician agreement on low symptom severity for $65 \%$ of patients, and there was agreement on high symptom severity for $11 \%$ of patients; however, for 3\% of patients, the clinicians rated symptom severity higher than their patients, and in turn, $21 \%$ of patients rated their constipation severity higher than their clinicians did.

Overall, there was moderate agreement $(\kappa=0.413-$ 0.570 ) on symptom severity for 9 of 17 symptoms (53\%), fair agreement on 7 symptoms ( $41 \%$; $\kappa=0.220-0.378)$, and slight agreement on 1 symptom $(6 \% ; \kappa=0.188)$. In the lower half of Table 2, we report comparisons of patient-reported symptom interference with clinicianreported severity. Again, we find moderate agreement $(\kappa=0.402-0.522)$ on 7 of 17 symptoms (41\%), fair 
TABLE 2. Agreement Between Patients and Clinicians: Maximum Symptom Severity and Interference Scores at Any Time During Treatment $(n=267)$

\begin{tabular}{|c|c|c|c|c|c|}
\hline \multirow[b]{2}{*}{ Symptom } & \multicolumn{4}{|c|}{ Agreement on Maximum Symptom Severity Score, \% } & \multirow[b]{2}{*}{$\mathrm{K}^{\mathrm{a}}$} \\
\hline & Agree Low & Agree High & Clinician High, Patient Low & Patient High, Clinician Low & \\
\hline Constipation & 65 & 11 & 3 & 21 & 0.329 \\
\hline Diarrhea & 62 & 14 & 4 & 21 & 0.378 \\
\hline Nausea & 65 & 14 & 7 & 14 & 0.437 \\
\hline Vomiting & 90 & 2 & 4 & 5 & 0.220 \\
\hline Mucositis, oral & 74 & 10 & 5 & 12 & 0.447 \\
\hline Fatigue, lack of energy & 31 & 48 & 7 & 14 & 0.570 \\
\hline Aching joints/arthralgia & 60 & 17 & 5 & 18 & 0.455 \\
\hline Aching muscles/myalgia & 62 & 13 & 6 & 20 & 0.363 \\
\hline Peripheral neuropathy & 60 & 16 & 11 & 13 & 0.413 \\
\hline Anxiety & 57 & 16 & 5 & 22 & 0.372 \\
\hline Feeling sad, unhappy/depression & 73 & 10 & 2 & 15 & 0.444 \\
\hline Insomnia & 43 & 27 & 5 & 25 & 0.416 \\
\hline Dyspnea/light-headedness & 77 & 4 & 4 & 15 & 0.224 \\
\hline Abdominal pain & 83 & 2 & 2 & 14 & 0.188 \\
\hline Edema, limbs & 79 & 4 & 1 & 17 & 0.245 \\
\hline General pain & 60 & 20 & 8 & 12 & 0.523 \\
\hline \multirow[t]{2}{*}{ Hot flashes } & 66 & 16 & 8 & 11 & 0.513 \\
\hline & \multicolumn{4}{|c|}{ Agreement on Maximum Symptom Interference Score, \% } & \\
\hline Symptom & Agree Low & Agree High & Clinician High, Patient Low & Patient High, Clinician Low & $\mathrm{K}^{\mathrm{a}}$ \\
\hline Constipation & 77 & 6 & 7 & 9 & 0.337 \\
\hline Diarrhea & 70 & 11 & 6 & 13 & 0.426 \\
\hline Nausea & 68 & 11 & 9 & 12 & 0.384 \\
\hline Vomiting & 89 & 1 & 4 & 6 & 0.150 \\
\hline Mucositis, oral & 80 & 6 & 9 & 6 & 0.348 \\
\hline Fatigue, lack of energy & 32 & 44 & 11 & 13 & 0.522 \\
\hline Aching joints/arthralgia & 64 & 14 & 9 & 14 & 0.402 \\
\hline Aching muscles/myalgia & 68 & 12 & 7 & 14 & 0.406 \\
\hline Peripheral neuropathy & 64 & 10 & 18 & 8 & 0.256 \\
\hline Anxiety & 68 & 13 & 9 & 11 & 0.441 \\
\hline Feeling sad, unhappy/depression & 79 & 8 & 4 & 9 & 0.506 \\
\hline Insomnia & 52 & 21 & 12 & 16 & 0.398 \\
\hline Dyspnea/light-headedness & 78 & 4 & 4 & 12 & 0.275 \\
\hline Abdominal pain & 85 & 3 & 1 & 11 & 0.265 \\
\hline Edema, limbs & 86 & 2 & 2 & 10 & 0.247 \\
\hline General pain & 61 & 18 & 11 & 10 & 0.466 \\
\hline Hot flashes & 71 & 8 & 16 & 5 & 0.311 \\
\hline
\end{tabular}

Low is defined as none or mild for patient-reported symptoms and as grade 0 or 1 for clinician-rated toxicities; high is defined as moderate, severe, or very severe for patient-reported symptoms and as grade 2,3 , or 4 for clinician-rated toxicities. For $\mathrm{k}$ interpretation, $<0.0$ is less than chance agreement, 0.01 to 0.20 is slight agreement, 0.21 to 0.40 is fair agreement, 0.41 to 0.60 is moderate agreement, 0.61 to 0.80 is substantial agreement, and 0.81 to 0.99 is almost perfect agreement. ${ }^{\mathrm{a}}$ All $\mathrm{k}$ values were statistically significant $(P \leq .05)$.

agreement on 9 symptoms $(53 \% ; \kappa=0.247-0.398)$, and slight agreement on 1 symptom $(6 \% ; \kappa=0.150)$. All $\kappa$ estimates were statistically significant $(P \leq .05)$.

\section{Variables Associated With Patient-Clinician Agreement on Symptom Severity Scores}

With 3 levels of agreement (agree, clinician high/patient low, and patient high/clinician low), associations with patient characteristics were explored (Supporting Table 2). The highest number of statistically significant associations (signifying differences between patient and clinician scores) was seen for race with respect to nausea $(P=.05)$, arthralgia $(P=.04)$, peripheral neuropathy $(P=.04)$, and dyspnea $(P=.05)$. These differences are further elucidated in Figure 3, which shows that in $21 \%$ of nonwhite patients and $10 \%$ of white patients, clinicians rated peripheral neuropathy severity low when patients rated it high $(P=.04)$. However, the reverse is shown for nausea, for which clinicians rated symptom severity low when patients rated it high in 15\% of white patients and $12 \%$ of nonwhite patients $(P=.05)$.

Similarly, Figure 3 presents significant differences by body mass index (BMI), with clinician severity scores for constipation lower than patient scores for patients with BMIs less than $25 \mathrm{~kg} / \mathrm{m}^{2}$ (14\%), BMIs of 25 to $30 \mathrm{~kg} / \mathrm{m}^{2}(35 \%)$, and BMIs of $30 \mathrm{~kg} / \mathrm{m}^{2}$ or higher $(17 \% ; P=.003)$. BMI-related differences are also shown for edema $(P=.005)$, with the rate of clinician 

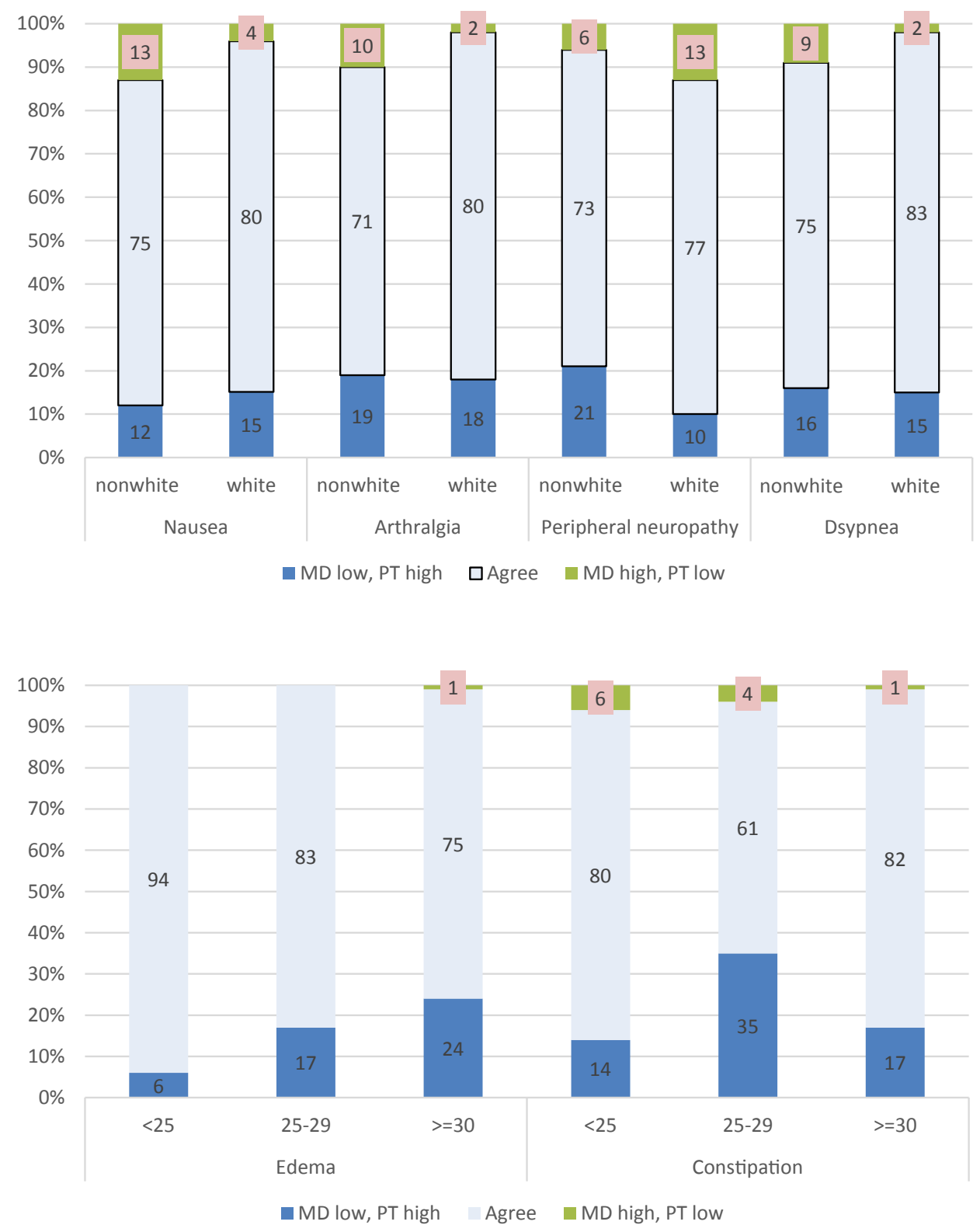

Figure 3. Patient (PT)-clinician (MD) congruence by race and body mass index (BMI).

underreporting increasing with increasing BMI levels. Regarding marital status, clinician severity scores for peripheral neuropathy were lower for unmarried patients $(19 \%)$ than married patients $(8 \% ; P=.03)$. There were no significant differences for age, education, or menopausal status.

\section{DISCUSSION}

Quality of life has been measured extensively in women with early breast cancer, ${ }^{27}$ but few studies have administered single-item symptom assessments related to specific treatment- or disease-related adverse events at frequent intervals during active treatment (which is an emerging standard for adverse event monitoring in clinical trials $)^{8}$ or have compared same-day patient and clinician reporting of this information. In our sample of women with early breast cancer, toxicity scores for 17 symptoms were collected longitudinally via single-item scales for patient-reported symptom severity and interference (PRO-CTCAE or PRSM) and clinician toxicity 
grades (CTCAE). Patients completed their form before seeing their oncologist, and clinicians completed their form after the visit. Scoring reports were not shared between patients and clinicians. The analysis was limited to patient-clinician scores that were collected on the same day ("paired").

Across all 17 symptoms, clinician toxicity grades were lower than patient-reported severity scores, as seen in the proportions of symptoms for which patients rated symptom severity high but clinicians rated toxicity low. This observation corroborates findings from an Italian study in women with early breast cancer that compared symptom questionnaires from patients at 2 time points (using a translation of CTCAE into Italian) with toxicity grades that were extracted and interpreted from clinician notes by research staff nurses. ${ }^{28}$ In our study, we note higher congruence between patients and clinicians when symptoms severity was low and lower congruence when symptom severity was high; this is similar to what has been previously reported in other studies. ${ }^{29}$ This observation is especially problematic when patients report high symptom severity but their clinicians note low toxicity, as observed for insomnia in $25 \%$ of patients, for anxiety in $22 \%$ of patients, for constipation in $25 \%$ of patients, for diarrhea in $21 \%$ of patients, and for myalgia in $20 \%$ of patients. It was exceptional when clinicians rated symptom toxicity high when their patients rated it low, as observed for peripheral neuropathy in $11 \%$ of patients, for general pain and hot flashes in $8 \%$ of patients, and for fatigue in $7 \%$ of patients.

We investigated patient-reported scores for "interference with what you usually like to do" and found them to be substantially lower than patient-reported symptom severity. We also compared patient-reported interference with clinician toxicity scores to see whether this comparison yielded greater congruence, but it did not. In our final analysis of the data, we found that patient characteristics were by and large not associated with patient-clinician disagreement on severity scores. However, we did find that clinician underestimation of certain symptoms was greater in nonwhite patients than white patients. This finding warrants further research but also reflects the larger literature documenting racial disparities in patient-provider communication ${ }^{30-33}$ as well as racial differences in symptom management experiences. ${ }^{34}$

We note that patients completed their form before seeing their oncologist. Clinicians completed their form after the visit but not always immediately after seeing the patient. It is possible that a substantial time lag (which we did not measure) between seeing the patient and completing the form may have affected a clinician's recall of the patient's symptom severity. We also did not gather data on whether the clinician form was completed by an MD, nurse practitioner, or physician assistant and, therefore, did not analyze potential differences among clinicians.

Patient-centered care, which is crucial to high-quality health care, ${ }^{35}$ requires the inclusion of the patient's assessment of treatment toxicity. It is important to understand when and how patient and clinician perspectives diverge and for which symptoms and patient characteristics they diverge. Our study points to the potential for racial disparities in symptom assessment by clinicians. The moderate or lower $\kappa$ agreement across all 17 symptoms suggests challenges in effective patient-clinician communication about symptom experience across domains of symptom clusters (eg, psychoneurological, gastrointestinal, and hormonal). ${ }^{36}$ Disagreement in scores tends to be at the high symptom severity end of the spectrum, with clinicians underestimating severity. Continuous symptom monitoring from both patients and clinicians, from before chemotherapy (to establish the patient's baseline) ${ }^{7}$ through the end of chemotherapy, provides an opportunity for early intervention for symptoms for which there are pharmaceutical remedies (eg, anxiety, depression, and insomnia) or nonpharmacological remedies (eg, moderate exercise to mitigate fatigue $\mathrm{3}^{37,38}$ ).

There is growing evidence that patients are willing and able to complete PRO-CTCAE items during a treatment-related clinic visit and after treatment has been completed. ${ }^{39}$ Using nurses and nurse navigators, clinics could consider developing processes to record and review patient-reported symptoms and consult with the oncologist for real-time interventions to reduce symptom severity. These processes would likely improve the likelihood of treatment completion, potentially improve patient quality of life during chemotherapy to the extent that toxicities are effectively managed, and enhance overall satisfaction with care. ${ }^{11,40}$ Alternative payment models for oncology could facilitate the incorporation of patientreported symptom assessment into quality metrics by providing reimbursement for these added responsibilities.

In conclusion, although clinician reporting of symptoms is common practice in oncology, there is suboptimal agreement with the gold standard of patient self-reporting, particularly for nonwhite patients. These data provide further evidence supporting the integration 
of patient-reported outcomes into cancer research and clinical practice to improve symptom monitoring and guide timely interventions. This, in turn, would enable the timely identification of symptoms for which there are evidence-based interventions. Our findings support attention to patient-clinician interactions that are patient-centered and focus on quality of life as well as effective symptom management with particular attention paid to cultural sensitivity. ${ }^{32,41}$ Further research is needed to explore approaches to encouraging and enabling patient-provider communication on symptom severity in ways that are actionable in real-world clinical practice.

\section{FUNDING SUPPORT}

This study was supported by the Breast Cancer Research Foundation (New York, New York), the Kay Yow Cancer Fund (Raleigh, North Carolina), and the University of North Carolina Lineberger Comprehensive Cancer Center/University Cancer Research Fund (Chapel Hill, North Carolina). The University of Texas MD Anderson Cancer Center is supported by the National Institutes of Health (grant P30 CA016672).

\section{CONFLICT OF INTEREST DISCLOSURES}

William A. Wood reports grants from Genentech and Pfizer outside the submitted work. The other authors made no disclosures.

\section{AUTHOR CONTRIBUTIONS}

Kirsten A. Nyrop: Primary authorship (original draft and review/editing of the article), conceptualization, and project administration. Allison M. Deal: Statistical analysis conceptualization and oversight. Bryce B. Reeve: Editing of the article and critical appraisal of the content. Ethan Basch: Editing of the article and critical appraisal of the content. Yi Tang Chen: Statistical analysis. Ji Hye Park: Statistical analysis. Shlomit S. Shachar: Verification of the methods, editing of the article, and critical appraisal of the content. Lisa A. Carey: Editing of the article and critical appraisal of the content. Katherine E. Reeder-Hayes: Editing of the article and critical appraisal of the content. Elizabeth C. Dees: Editing of the article and critical appraisal of the content. Trevor A. Jolly: Editing of the article and critical appraisal of the content. Gretchen G. Kimmick: Editing of the article and critical appraisal of the content. Meghan S. Karuturi: Editing of the article and critical appraisal of the content. Raquel E. Reinbolt: Editing of the article and critical appraisal of the content. JoEllen C. Speca: Editing of the article and critical appraisal of the content. Jordan T. Lee: Data collection, editing of the article, and critical appraisal of the content. William A. Wood: Editing of the article and critical appraisal of the content. Hyman B. Muss: Senior authorship, contributions to the original draft and review/editing of the article, verification of the methods, and critical appraisal of the content.

\section{REFERENCES}

1. National Cancer Institute. Common Terminology Criteria for Adverse Events (CTCAE) v5.0. https://ctep.cancer.gov/protocolDevelopment/ electronic_applications/ctc.htm\#ctc_50

2. Velikova G, Booth L, Smith AB, et al. Measuring quality of life in routine oncology practice improves communication and patient wellbeing: a randomized controlled trial. J Clin Oncol. 2004;22:714-724.

3. Atkinson TM, Ryan SJ, Bennett AV, et al. The association between clinician-based Common Terminology Criteria for Adverse Events (CTCAE) and patient-reported outcomes (PRO): a systematic review. Support Care Cancer. 2016;24:3669-3676.

4. Basch E, Reeve BB, Mitchell SA, et al. Development of the National Cancer Institute's Patient-Reported Outcomes Version of the Common
Terminology Criteria for Adverse Events (PRO-CTCAE). J Natl Cancer Inst. 2014;106:dju244.

5. Dueck AC, Mendoza TR, Mitchell SA, et al. Validity and reliability of the US National Cancer Institute's Patient-Reported Outcomes Version of the Common Terminology Criteria for Adverse Events (PROCTCAE). JAMA Oncol. 2015;1:1051-1059.

6. Kluetz PG, Kanapuru B, Lemery S, et al. Informing the tolerability of cancer treatments using patient-reported outcome measures: summary of an FDA and Critical Path Institute Workshop. Value Health. 2018;21:742-747.

7. Pe M, Dorme L, Coens C, et al. Statistical analysis of patientreported outcome data in randomised controlled trials of locally advanced and metastatic breast cancer: a systematic review. Lancet Oncol. 2018;19:e459-e469.

8. Basch E, Bennett A, Pietanza MC. Use of patient-reported outcomes to improve the predictive accuracy of clinician-reported adverse events. J Natl Cancer Inst. 2011;103:1808-1810.

9. Falchook AD, Green R, Knowles ME, et al. Comparison of patientand practitioner-reported toxic effects associated with chemoradiotherapy for head and neck cancer. JAMA Otolaryngol Head Neck Surg. 2016;142:517-523.

10. Basch E, Iasonos A, McDonough T, et al. Patient versus clinician symptom reporting using the National Cancer Institute Common Terminology Criteria for Adverse Events: results of a questionnaire-based study. Lancet Oncol. 2006;7:903-909.

11. Basch E, Jia X, Heller G, et al. Adverse symptom event reporting by patients vs clinicians: relationships with clinical outcomes. J Natl Cancer Inst. 2009;101:1624-1632.

12. Moon DH, Chera BS, Deal AM, et al. Clinician-observed and patient-reported toxicities and their association with poor tolerance to therapy in older patients with head and neck or lung cancer treated with curative radiotherapy. J Geriatr Oncol. 2019;10:42-47.

13. Laugsand EA, Sprangers MA, Bjordal K, et al. Health care providers underestimate symptom intensities of cancer patients: a multicenter European study. Health Qual Life Outcomes. 2010;8:104.

14. Fromme EK, Eilers KM, Mori M, et al. How accurate is clinician reporting of chemotherapy adverse effects? A comparison with patient-reported symptoms from the Quality-of-Life Questionnaire C30. J Clin Oncol. 2004;22:3485-3490.

15. Quinten C, Maringwa J, Gotay CC, et al. Patient self-reports of symptoms and clinician ratings as predictors of overall cancer survival. J Natl Cancer Inst. 2011;103:1851-1858.

16. Cirillo M, Venturini M, Ciccarelli L, et al. Clinician versus nurse symptom reporting using the National Cancer Institute-Common Terminology Criteria for Adverse Events during chemotherapy: results of a comparison based on patient's self-reported questionnaire. Ann Oncol. 2009;20:1929-1935.

17. Nyrop KA, Deal AM, Shachar SS, et al. Patient-reported toxicities during chemotherapy regimens in current clinical practice for early breast cancer. Oncologist. 2019;24:762-771.

18. Nyrop KA, Deal AM, Reeder-Hayes KE, et al. Patient and clinicianreported chemotherapy-induced peripheral neuropathy (CIPN) in early breast cancer: current clinical practice. Cancer. 2019;125:2945-2954.

19. Stover A, Irwin DE, Chen RC, et al. Integrating patient-reported outcome measures into routine cancer care: cancer patients' and clinicians' perceptions of acceptability and value. EGEMS (Wash DC). 2015;3:1169.

20. Hay JL, Atkinson TM, Reeve BB, et al. Cognitive interviewing of the US National Cancer Institute's Patient-Reported Outcomes Version of the Common Terminology Criteria for Adverse Events (PROCTCAE). Qual Life Res. 2014;23:257-269.

21. Atkinson TM, Hay JL, Dueck AC, et al. What do "none," "mild," "moderate," "severe," and "very severe" mean to patients with cancer? Content validity of PRO-CTCAE response scales. J Pain Symptom Manage. 2018;55:e3-e6.

22. Basch E, Becker C, Rogak LJ, et al. Development of a composite scoring algorithm for the National Cancer Institute's Patient-Reported Outcomes Version of the Common Terminology Criteria for Adverse Events (PRO-CTCAE). Paper presented at: 26th Annual Meeting of the International Society for Quality of Life Research; October 20-23, 2019; San Diego, CA. 
23. Basch E, Rogak LJ, Dueck AC. Methods for implementing and reporting patient-reported outcome (PRO) measures of symptomatic adverse events in cancer clinical trials. Clin Ther. 2016;38:821-830.

24. Basch E, Artz D, Dulko D, et al. Patient online self-reporting of toxicity symptoms during chemotherapy. J Clin Oncol. 2005;23:3552-3561.

25. Altman DG. Practical Statistics for Medical Research. Chapman and Hall; 1991.

26. Landis JR, Koch GG. The measurement of observer agreement for categorical data. Biometrics. 1977;33:159-174.

27. Lemieux J, Goodwin PJ, Bordeleau LJ, et al. Quality-of-life measurement in randomized clinical trials in breast cancer: an updated systematic review (2001-2009). J Natl Cancer Inst. 2011;103:178-231.

28. Montemurro F, Mittica G, Cagnazzo C, et al. Self-evaluation of adjuvant chemotherapy-related adverse effects by patients with breast cancer. JAMA Oncol. 2016;2:445-452.

29. Atkinson TM, Reeve BB, Dueck AC, et al. Application of a Bayesian graded response model to characterize areas of disagreement between clinician and patient grading of symptomatic adverse events. J Patient Rep Outcomes. 2018;2:56.

30. Palmer NR, Kent EE, Forsythe LP, et al. Racial and ethnic disparities in patient-provider communication, quality-of-care ratings, and patient activation among long-term cancer survivors. J Clin Oncol. 2014;32:4087-4094.

31. Royak-Schaler R, Passmore SR, Gadalla S, et al. Exploring patientphysician communication in breast cancer care for African American women following primary treatment. Oncol Nurs Forum. 2008;35:836-843.

32. Gonzales FA, Sangaramoorthy M, Dwyer LA, et al. Patient-clinician interactions and disparities in breast cancer care: the Equality in Breast Cancer Care Study. J Cancer Surviv. 2019;13:968-980.

33. Robertson-Jones TA, Tissue MM, Connolly M, Gallups SF, Bender $\mathrm{CM}$, Rosenzweig MQ. Exploring racial differences in patient centeredness of care (PCC) during breast cancer (BC) chemotherapy clinical visits. J Racial Ethn Health Disparities. 2019;6:94-100.

34. Samuel CA, Schaal J, Robertson L, et al. Racial differences in symptom management experiences during breast cancer treatment. Support Care Cancer. 2018;26:1425-1435.

35. Institute of Medicine Committee on Quality of Health Care in America. Crossing the Chasm: A New Health System for the 21st Century. National Academy Press; 2001.

36. Kim HJ, Barsevick AM, Tulman L, et al. Treatment-related symptom clusters in breast cancer: a secondary analysis. J Pain Symptom Manage. 2008;36:468-479.

37. Brown JC, Huedo-Medina TB, Pescatello LS, et al. Efficacy of exercise interventions in modulating cancer-related fatigue among adult cancer survivors: a meta-analysis. Cancer Epidemiol Biomarkers Prev. 2011;20:123-133.

38. Cramp F, Byron-Daniel J. Exercise for the management of cancerrelated fatigue in adults. Cochrane Database Syst Rev. 2012;11: CD006145.

39. Basch E, Pugh SL, Dueck AC, et al. Feasibility of patient reporting of symptomatic adverse events via the Patient-Reported Outcomes Version of the Common Terminology Criteria for Adverse Events (PRO-CTCAE) in a Chemoradiotherapy Cooperative Group multicenter clinical trial. Int J Radiat Oncol Biol Phys. 2017;98:409-418.

40. Neugut AI, Hillyer GC, Kushi LH, et al. A prospective cohort study of early discontinuation of adjuvant chemotherapy in women with breast cancer: the Breast Cancer Quality of Care Study (BQUAL). Breast Cancer Res Treat. 2016;158:127-138.

41. Check DK, Chawla N, Kwan ML, et al. Understanding racial/ethnic differences in breast cancer-related physical well-being: the role of patient-provider interactions. Breast Cancer Res Treat. 2018;170: 593-603. 